\title{
Guest editor's preface
}

The eleventh IFIP database security conference was held on 7-9 August 1997 at Lake Tahoe, California. All the presentations are collected in the book "Database Security: Status and Prospect Volume 11" and published in early 1998. However, due to space limitations, each paper was limited to 15 pages. By the invitation of the Editor-in-Chief, Professor S. Jajodia, of the Journal of Computer Security, this guest editor invited authors to re-write their presentations in full length for journal articles. Through the referee process, 6 papers were selected.

Purely based on space considerations, they are divided into two issues. The first issue consists of three papers. In alphabetical order by first authors, the first paper selected is "Secure object deletion and garbage collection in multilevel object bases" by Elisa Bertino and Elena Ferrari. The paper discusses in depth the problem of secure object deletion in Object Database Management Systems. The second paper is "Modelling, specifying and implementing workflow security in Cyberspace" by Ehud Gudes, Martin S. Olivier and Reind P. van de Riet. The authors present an orderly framework to model security issues related to workflow systems, to specify security requirements within this model, and then to map such specifications to the implementation level. The final selection is by Ravi Sandhu and Venkata Bhamidipati. Their paper "Role-based administration of user-role assignment: The URA97 model and its Oracle implementation" introduces a role-based decentralized administrative model for assigning users to roles, and describes an implementation of this model on the Oracle platform.

In the second issue the following three papers will be published: "A semanticbased execution model for multilevel secure workflows" by Vijayalakshmi Atluri, Wei-Kuang Huang and Elisa Bertino, "A ticket-based access control architecture for object systems" by John Hale, Jody Threet and Sujeet Shenoi, and "Structured namespaces in secure databases" by Adrian Spalka and Armin B. Cremers. Regrettably, space limitation precluded several equally interesting papers presented in the conference from being included in these two issues.

T.Y. Lin

San José State University 